\title{
Readmissions and clinical outcomes in heart failure patients: A retrospective study
}

\author{
Sai Sree Lekha Akkineni ${ }^{\mathrm{a}}$, Omair Mohammed ${ }^{\mathrm{a}}$, John Preshanth Kumar Pathiraj ${ }^{\mathrm{a}}$, Tom Devasia ${ }^{\mathrm{b}}$, \\ Ramesh Chandrababuc ${ }^{c}$, Vijayanarayana Kunhikatta ${ }^{\mathrm{a}, *}$ \\ ${ }^{a}$ Department of Pharmacy Practice, Manipal College of Pharmaceutical Sciences, Manipal Academy Higher Education, Manipal, 576104, Karnataka State, India \\ ${ }^{\mathrm{b}}$ Department of Cardiology, Kasturba Medical College, Manipal, Manipal Academy Higher Education, Manipal, 576104, Karnataka State, India \\ ${ }^{\mathrm{c}}$ Department of Medical Surgical Nursing, Manipal College of Nursing, Manipal Academy Higher Education, Manipal, 576104, Karnataka State, India
}

\section{A R T I C L E I N F O}

\section{Keywords:}

Heart failure

Risk factors

Readmissions

In-hospital mortality

\begin{abstract}
A B S T R A C T
Background: Heart failure is a syndrome, due to structural and/or functional abnormalities of the heart associated with high mortality and readmissions. Causes of readmissions are poorly understood and identifying the factors associated with readmissions are crucial to reduce poor clinical outcomes in these patients.

Objectives: The main objective of this study was to identify the factors associated with readmissions and clinical improvement in heart failure patients.

Materials and methods: A case-control study was conducted in South Indian tertiary care teaching hospital from August 2017 to February 2018. Heart failure patients with readmissions (cases) and without readmissions (control) were identified from medical records department patient files using International Classification of Diseases 10 code 150. Patient's demographics, medical history, laboratory parameters, treatment pattern, readmissions and clinical outcome were collected. Factors associated with readmissions and improved clinical outcome were identified by logistic regression.

Results: Out of the total 451 heart failure patients $63.6 \%$ were male. The mean age of the study population was $59.7 \pm 14.1$. Hypothyroidism (OR: 2.64; $\mathrm{p}=0.041$ ), diabetes mellitus (OR: $1.59 ; \mathrm{p}=0.019$ ) and hypertension (OR: 1.53; $\mathrm{p}<0.034$ ) were identified as statistically significant factors associated with readmissions. Conversion of intravenous to per oral diuretics (OR: 1.98; $\mathrm{p}=0.019$ ) and ejection fraction at readmission (OR: 1.04; $\mathrm{p}=0.020$ ) were associated with clinical improvement.

Conclusions: Comorbidities like hypothyroidism, diabetes mellitus and hypertension were associated with increased readmissions in patients with heart failure in this study.
\end{abstract}

\section{Introduction}

Heart failure (HF) has emerged as one of the leading causes of hospitalization and mortality worldwide, with the prevalence of over 26 million. ${ }^{1}$ There is an estimated prevalence of 5 and 15 million in the United States and European countries respectively. ${ }^{1}$ India has an estimated prevalence of about 1.3-2.3 million. ${ }^{2}$ American Heart Association reports that the lifetime risk of developing HF in men and women at the age of 40 is 1 in $5{ }^{1}$ Probable etiologies include coronary artery disease, previous episode of myocardial infarction, hypertension, diabetes mellitus, abnormal heart valves (either congenital or disease oriented). Other secondary causes include severe anemia, thyroid disorders, dysrhythmia, obesity, sleep apnea and chronic lung disease.

The prevalence of HF has been on the rise in almost all the developed as well as developing countries due to increase in the elderly population, betterment in the efficacy of treatment options available etc. One in nine deaths has HF referenced on the death certificate. ${ }^{3}$

Hospitalizations are common after HF diagnosis, with $83 \%$ patients hospitalized atleast once and $43 \%$ hospitalized atleast 4 times. ${ }^{3} \mathrm{~A}$ number of therapies have been developed over the last 2 decades which claim to reduce hospitalizations due to exacerbation of symptoms due to heart failure with quality improvement initiatives being developed. ${ }^{4}$

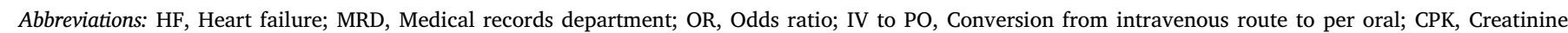

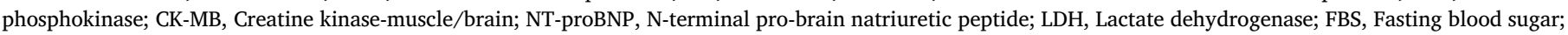
T.Ch, Total cholesterol; LDL, Low density lipoprotein; BMI, Body mass index

* Corresponding author.

E-mail address: vijayanarayana.k@manipal.edu (V. Kunhikatta). 


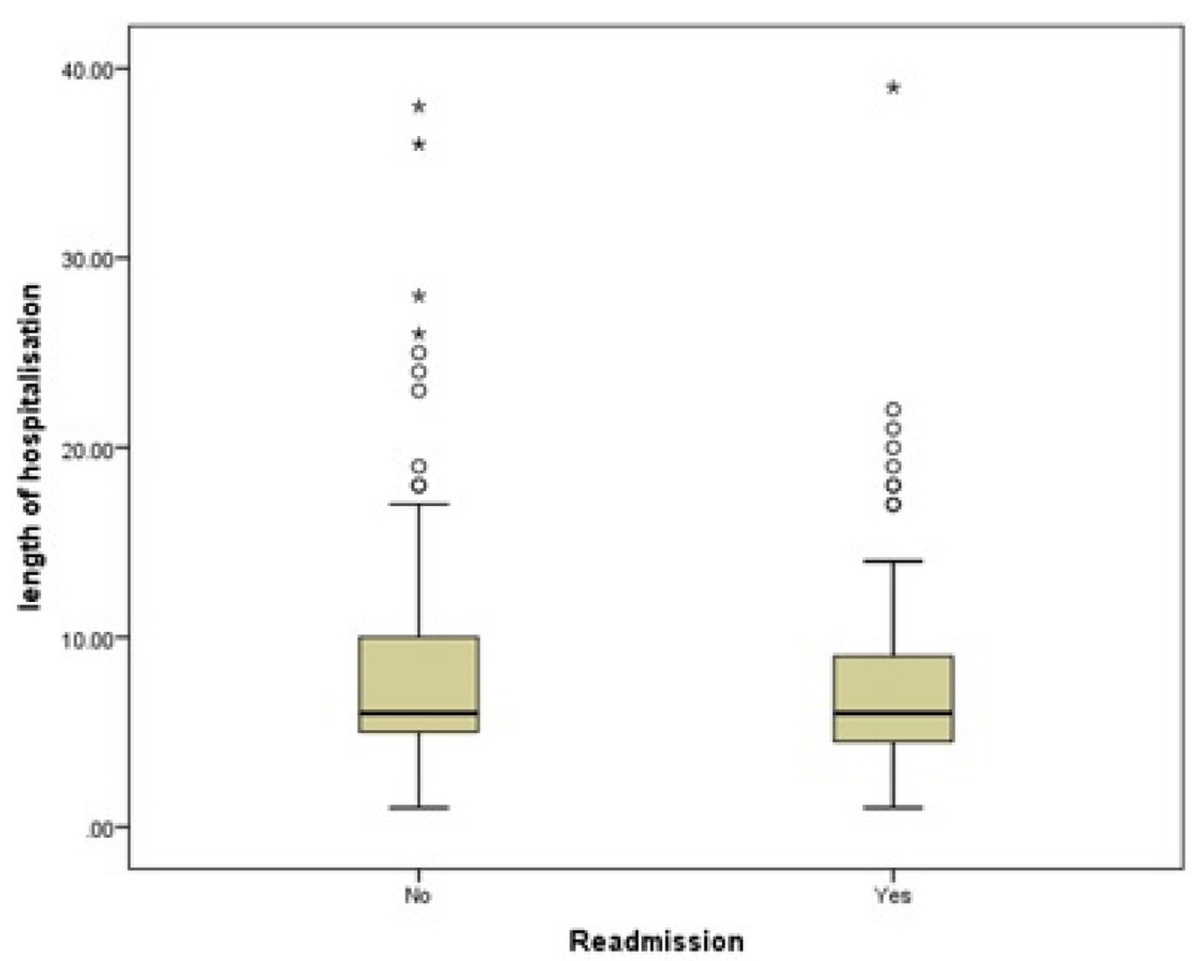

Fig. 1. Length of hospitalization days in HF patient with readmission and no readmissions.

But in the long run, none of them have been shown to significantly reduce the multiple readmissions and mortality rate leading to a substantial socio-economic burden, loss of productivity and a decrease in quality of life scores. Identifying predictors for repeat hospitalizations or poorer outcome among HF patients could help physicians to risk stratify patients and determine optimal post discharge plan.

Objectives: The objectives of this study were to identify the factors associated with readmissions and clinical improvement in heart failure patients.

\section{Materials and methods}

\subsection{Study design}

Hospital based case-control study.

\subsection{Setting}

Study was conducted in a tertiary care teaching hospital, during August 2017 to March 2018, after obtaining Institutional Ethics Committee approval.

\subsection{Participants}

In-patients diagnosed with heart failure as a primary disease from 1st January 2012 to 31st December 2016 and in compliance with the inclusion and exclusion criteria were enrolled in the study. The subjects were identified using the ICD 10 code 150 from medical records department (MRD). Criteria followed for inclusion were as mentioned below:

- Case: Patients who were readmitted atleast once who were diagnosed with HF during index hospitalization.

- Control: HF patients who were not readmitted following an index hospitalization.

- Readmission: Cases who were re-hospitalized with cardiac associated symptoms like dyspnea, pedal edema, chest pain post discharge from index hospitalization.

- Clinical improvement: Symptoms improvement clinical diagnosed by atleast once functional class according to New York Heart Association (NYHA).

\subsection{Inclusion and exclusion criteria}

The inclusion criteria in-patients with a definitive diagnosis of HF. We excluded out-patients, patients with end stage disease of the kidney, liver and/or lung developed prior to the diagnosis of HF, patients with HF but who are now/then being admitted with other diagnoses, patients with incomplete records.

\subsection{Data collection}

Pertinent information was collected from the MRD. Variables included demographics, medical and medication history, laboratory parameters, treatment and progress charts, readmission and clinical outcome data which was recorded on the pre-designed case record form.

\subsection{Statistical analyses}

Parametric data were presented as mean \pm SD, skewed and nonparametric data were expressed with mean \pm SD and range Nominal data were presented as frequency and percentage. Independent sample $t$-test, Mann-Whitney or Chi square test was performed to compare the parameters between HF patients with readmissions and without readmissions. Identification factors associated with readmissions and clinical improvement and calculation of odds ratio (OR) was done by logistic regression. A p value of $<0.05$ was considered statistically significant. Data entry and statistical analysis was done using IBM SPSS Statistics for version 20.0 (IBM Corp. Armonk, NY). 
Table 1

Demographic and biochemical parameters of HF patients with readmissions and without readmissions.

\begin{tabular}{|c|c|c|c|c|}
\hline Parameter & All HF patients $(n=451)$ & HF patients with Readmissions $(\mathrm{n}=159)$ & HF patients without Readmissions $(n=292)$ & p- value \\
\hline Age in years (mean $\pm S D$ ) & $59.70 \pm 14.10$ & $60.14 \pm 12.20$ & $59.56 \pm 15.00$ & 0.659 \\
\hline \multicolumn{5}{|l|}{ Gender } \\
\hline Male, n (\%) & $287(63.6)$ & $107(67.3)$ & $180(61.6)$ & \multirow[t]{2}{*}{0.225} \\
\hline Female, n (\%) & $164(36.4)$ & $52(32.7)$ & $112(38.4)$ & \\
\hline No of readmissions (mean \pm SD \& Range) & - & $2.11 \pm 2.35^{(1-19)}$ & - & - \\
\hline Ejection fraction in $\%$ (Mean \pm SD) & $40.3 \pm 3.80$ & $40.38 \pm 2.10$ & $40.37 \pm 4.30$ & 0.993 \\
\hline $\mathrm{CPK}$ in $\mathrm{IU} / \mathrm{L}($ mean $\pm \mathrm{SD})$ & $320.68 \pm 41.91$ & $402.78 \pm 52.54$ & $272.66 \pm 81.67$ & 0.373 \\
\hline Troponin $\mathrm{T}$ in $\mathrm{ng} / \mathrm{mL}$ (mean $\pm \mathrm{SD}$ ) & $0.57 \pm 0.16$ & $1.18 \pm 0.22$ & $0.26 \pm 0.17$ & 0.194 \\
\hline $\mathrm{CK}-\mathrm{MB}$ in $\mu \mathrm{g} / \mathrm{L}($ mean $\pm \mathrm{SD})$ & $10.58 \pm 2.44$ & $11.35 \pm 3.74$ & $8.84 \pm 2.41$ & 0.691 \\
\hline NT-proBNP in $\mathrm{pg} / \mathrm{mL}$ (mean $\pm \mathrm{SD}$ ) & $12903.31 \pm 3250.87$ & $14921 \pm 3933.14$ & $8931.80 \pm 871.37$ & 0.235 \\
\hline LDH in Units/L (mean \pm SD) & $518.87 \pm 37.14$ & $442.60 \pm 413.82$ & $533.55 \pm 675.95$ & 0.742 \\
\hline Creatinine in $\mathrm{mg} / \mathrm{dL}$ (mean $\pm \mathrm{SD}$ ) & $1.31 \pm 0.90$ & $1.32 \pm 1.08$ & $1.31 \pm 0.79$ & 0.930 \\
\hline Potassium in $\mathrm{mEq} / \mathrm{L}($ mean $\pm \mathrm{SD})$ & $5.72 \pm 1.65$ & $6.34 \pm 1.25$ & $4.61 \pm 0.85$ & $0.029^{*}$ \\
\hline FBS in $\mathrm{mg} / \mathrm{dL}($ mean $\pm \mathrm{SD})$ & $139.42 \pm 71.73$ & $142.90 \pm 71.33$ & $136.87 \pm 72.16$ & 0.517 \\
\hline T.Ch in $\mathrm{mg} / \mathrm{dL}$ (mean $\pm \mathrm{SD}$ ) & $138.64 \pm 46.28$ & $141.97 \pm 46.08$ & $136.42 \pm 46.46$ & 0.396 \\
\hline $\mathrm{LDL}$ in $\mathrm{mg} / \mathrm{dL}($ mean $\pm \mathrm{SD})$ & $85.22 \pm 37.13$ & $86.23 \pm 37.09$ & $83.69 \pm 37.38$ & 0.631 \\
\hline $\mathrm{BMI}$ in $\mathrm{kg} / \mathrm{m}^{2}($ mean $\pm \mathrm{SD})$ & $23.32 \pm 5.89$ & $23.31 \pm 5.45$ & $23.34 \pm 6.19$ & 0.972 \\
\hline Unimproved, n (\%) & $64(14.1)$ & $20(12.6)$ & $44(15.1)$ & 0.470 \\
\hline
\end{tabular}

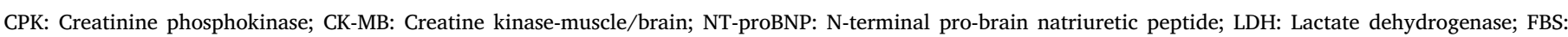
Fasting blood sugar; T.Ch: Total cholesterol; LDL: Low density lipoprotein; BMI: Body mass index.

*Statistically significant compared to HF patients without readmissions.

\section{Results}

This study included index admissions of 451 patients who met the inclusion and exclusion criteria. Out of $451 \mathrm{HF}$ patients 159 had one or more readmissions related to symptoms or complications of HF during the follow-up period. Mean age of the HF patients with readmissions was $60.14 \pm 12.2$ years compared to $59.56 \pm 15.0$ years in HF patients without readmissions. Average number of readmissions in $\mathrm{HF}$ patients with readmission was $2.11 \pm 2.35$ (range $=1-19$ ). Median days of hospitalization at index event in HF patients with readmissions was 6 with IQR $=4-9$, see Fig. 1 . Majority of the HF patients with readmissions were male (67.3\%). HF patients with readmissions had statistically significant (at $\mathrm{p}=0.029$ ), but moderate hyperkalemia compared to HF patients without readmissions. Though biochemical markers like Creatinine phosphokinase (CPK), troponin T, NT-proBNP and fasting blood sugar (FBS) were higher than normal value in HF patients with readmissions compared to without readmissions, but they were not statistically significant. Among readmission group, $12.6 \%$ $(\mathrm{n}=20)$ of patients were clinically improved. Demographic and biochemical parameters of HF patients with readmissions and without readmissions are given in Table 1.

Anemia (52.2\%) was the most common co-morbidity followed by diabetes mellitus (51.6\%) and hypertension (49.1\%) among HF patients with readmissions. Hypothyroidism was present among $6.9 \%$ of the HF patients with readmissions. Pulmonary hypertension was one of the complications and was seen in $78 \%$ of the HF patients with readmissions.

Dyspnea and breathlessness (88.1\%) were the most common clinical symptoms present in HF patients with readmissions. See Table 2.

Factors affecting readmissions in HF patients were analysed by logistic regression and odds ratio was calculated. Hypothyroidism (OR: 2.64; 95\% CI: 1.04-6.70; $\mathrm{p}=0.041$ ), diabetes mellitus (OR: 1.59; 95\% CI: $1.08-2.35 ; \mathrm{p}=0.019$ ) and hypertension (OR: $1.53 ; 95 \% \mathrm{CI}$ :
Table 3

Factors affecting readmissions in HF patients.

\begin{tabular}{llllll}
\hline Factors & $\begin{array}{l}\text { HF patients } \\
\text { with } \\
\text { Readmissions } \\
(\mathrm{n}=159)\end{array}$ & $\begin{array}{l}\text { HF patients } \\
\text { without } \\
\text { Readmissions } \\
(\mathrm{n}=292)\end{array}$ & OR & $95 \% \mathrm{CI}$ & p- value \\
& $107(67.3)$ & $180(61.6)$ & 1.28 & $0.86-1.92$ & 0.225 \\
\hline $\begin{array}{l}\text { Male Gender, n } \\
\quad \%)\end{array}$ & $1.18 \pm 6.22$ & $0.26 \pm 0.67$ & 1.09 & $0.95-1.26$ & 0.221 \\
$\begin{array}{l}\text { Troponin T } \\
\quad \text { (mean } \pm \text { SD) }\end{array}$ & & & & & \\
Potassium & $4.61 \pm 0.85$ & $6.34 \pm 13.25$ & 0.96 & $0.89-1.03$ & 0.281 \\
Hypothyroidism & $11(6.9)^{*}$ & $8(2.7)$ & 2.64 & $1.04-6.70$ & $0.041^{*}$ \\
DM & $82(51.6)^{*}$ & $117(40.1)$ & 1.59 & $1.08-2.35$ & $0.019^{*}$ \\
Hypertension & $78(49.1)^{*}$ & $113(38.7)$ & 1.53 & $1.03-2.25$ & $0.034^{*}$ \\
Atrial fibrillation & $7(4.4)$ & $24(8.2)$ & 0.51 & $0.22-1.22$ & 0.132 \\
Anemia & $83(52.2)$ & $170(58.2)$ & 1.30 & $0.88-1.88$ & 0.219 \\
Pulmonary HT & $124(77.9)$ & $213(72.9)$ & 1.30 & $0.83-2.07$ & 0.240 \\
ARF/CRF & $13(8.2)$ & $38(13.0)$ & 0.59 & $0.31-1.15$ & 0.124 \\
PDE inhibitors & $21(13.2)$ & $27(9.2)$ & 1.49 & $0.82-2.74$ & 0.195 \\
Diuretics & $125(78.6)$ & $210(79.1)$ & 1.44 & $0.91-2.27$ & 0.121 \\
Diuretic IV to PO & $117(73.6)$ & $231(79.1)$ & 0.74 & $0.47-1.16$ & 0.183 \\
\hline
\end{tabular}

OR: Odds ratio; CI: Confidence interval; DM: Diabetes mellitus; Pulmonary HT: Pulmonary hypertension; ARF/CRF: Acute renal failure/Chronic renal failure; PDE: Phosphodiesterase; IV to PO: Conversion from intravenous route to per oral.

* Statistically significant compared HF patients without readmissions; data was analysed by logistic regression.

1.03-2.25; $\mathrm{p}<0.034$ ) were identified as statistically significant factors associated with readmissions in HF patients. Results of logistic regression is shown in Table 3.

Factors associated with clinical improvement in HF patients were analysed by logistic regression and results are given in Table 4 . Conversion of IV (intravenous) to PO (per oral) diuretics (OR:1.98; 95\% CI: $1.12-3.50 ; \mathrm{p}=0.019$ ) and ejection fraction at readmission (OR: 1.04;

Table 2

Clinical signs and symptoms of HF patients with readmissions and without readmissions.

\begin{tabular}{|c|c|c|}
\hline Sign and Symptoms & HF patients with Readmissions $(\mathrm{n}=159)$ & HF patients without Readmissions $(n=292)$ \\
\hline Dyspnea \& breathlessness, n (\%) & $140(88.1)$ & $193(66.1)$ \\
\hline Pedal edema, $n(\%)$ & $58(36.5)$ & $130(44.5)$ \\
\hline Elevated JVP, n (\%) & $45(28.3)$ & $100(34.2)$ \\
\hline
\end{tabular}

JVP: Jugular venous pressure. 
Table 4

Factors associated with clinical improvement in heart failure patients.

\begin{tabular}{|c|c|c|c|c|c|}
\hline Factors & Symptoms Improved $(\mathrm{n}=387)$ & Symptoms Unimproved $(n=64)$ & OR & $95 \% \mathrm{CI}$ & p- value \\
\hline Diuretic IV to PO, n (\%) & $306(79.1)$ & $42(65.6)$ & 1.98 & $1.12-3.50$ & $0.019 *$ \\
\hline $\mathrm{EF}$ in $\%($ mean $\pm \mathrm{SD})$ & $41.21 \pm 13.55$ & $35.29 \pm 12.18$ & 1.04 & $1.01-1.07$ & $0.020 *$ \\
\hline Pulmonary HT, n (\%) & $283(73.1)$ & $54(84.4)$ & 0.50 & $0.25-1.03$ & $0.050 * *$ \\
\hline Globulin in $\mathrm{g} / \mathrm{dL}$ (mean $\pm \mathrm{SD}$ ) & $4.90 \pm 3.06$ & $11.40 \pm 3.36$ & 0.66 & $0.44-0.97$ & $0.034 * *$ \\
\hline $\mathrm{ARB}, \mathrm{n}(\%)$ & $62(16.0)$ & $6(9.4)$ & 1.84 & $0.76-4.46$ & 0.174 \\
\hline ACE inhibitor, n (\%) & $129(33.3)$ & $18(28.1)$ & 1.28 & $0.71-2.29$ & 0.411 \\
\hline
\end{tabular}

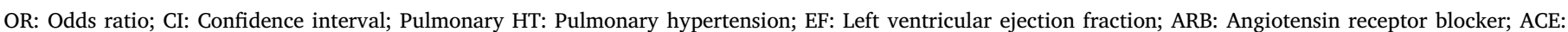
Angiotensin converting enzyme.

* Statistically significant compared HF patients who have not improved; data was analysed by logistic regression.

**Statistically significant compared HF patients who have improved; data was analysed by logistic regression.

95\% CI: $1.01-1.07 ; \mathrm{p}=0.020$ ) were statistically significant factors associated with clinical improvement, whereas pulmonary hypertension (OR: 0.50; 95\%: 0.25-1.03; $\mathrm{p}=0.050$ ) and plasma globulin level (OR: 0.66 ; $95 \%$ CI: $0.44-0.97 ; p=0.034$ ) were associated with poor clinical outcome. Though the use of ARBs and ACE inhibitors were associated with clinical improvements, they were not statistically significant.

However, use of ACE inhibitor was shown to reduce more than 1 readmission's (OR: 0.54; 95\% CI: 0.29-0.99; $\mathrm{p}=0.047$ ).

\section{Discussion}

Heart failure resulting in hospitalization represents a significant and growing health care burden. On hospitalization, a vast majority of patients symptomatically improve. However, their early post-discharge rehospitalization and mortality rates continue to be high. Worsening signs and symptoms, neurohormonal and renal abnormalities occurring soon after discharge may contribute to these high event rates. Further investigation into pathophysiologic targets and novel approaches to treatment designs are needed. The present study was aimed at estimating the factors associated with readmission and factors that contribute to improvement in clinical outcomes in a retrospective cohort.

Of the total 451 study patients, male predominance of $63.6 \%$ with a mean age of $59.76 \pm 14.11$ and a median hospital stay of $6 \pm 4$ days corroborating a similar previously reported finding. ${ }^{5,6}$ The mean number of days for which the patients were experiencing symptoms before hospital admission was $10.77 \pm 17.6$. Jia rong wu et al. reported patients who waited due to severe dyspneic symptoms before seeking consultation resulted in prolonged admission and exacerbation of $\mathrm{HF}^{7}$ suggesting greater the wait in number of days to consult the physician, higher the risk of readmission and worsened clinical outcome.

Several comorbidities were associated with the increase in the number of readmissions HF patients which is in agreement with previously published studies of which Diabetes Mellitus, Hypertension and Hypothyroidism remained significant. ${ }^{8-11}$ This suggests the importance of attaining optimum therapeutic outcomes of these risk factors/comorbidities and effective monitoring during the disease course. Restoration of euthyroidism normalized cardiac function shortening the iso-volumic relaxation time with an increase of early diastolic mitral flow velocity ratio, and increased left ventricular ejection fraction on exertion (results not shown).

Hyperkaliemia, though found to be significant parameter in the readmission group possibly attributed to the adverse effects of ACE inhibitor and potassium sparing diuretics could be potential confounder due to its presence in both group which could also be neutralized by loop diuretics.

Administration of loop diuretics showed significant improvement of symptom, reiterating its beneficial therapeutic effects hence being the mainstay for decongestion in acute HF when included initially. ${ }^{11}$ In spite of their clinical benefits, non-potassium-sparing diuretics may cause further neurohormonal activation and acute decompensation secondary to renal abnormalities. Patients who were switched from IV to oral diuretics showed improved clinical and in-hospital outcome than otherwise. Ejection fraction a diagnostic and a prognostic marker for systolic HF was seen to be improved in patients who were reported to show symptom improvement than those who did not. ${ }^{12}$ This could be attributed to suboptimal pharmaceutical care and medical management during their previous discharge.

Another prognostic ECHO finding, a complication of HF which is reported to result in poor outcomes and was statistically significant was pulmonary hypertension (PHTN) ${ }^{13}$ in the symptoms improved group suggesting volume and pressure overload, necessitating themselves for hospitalization and candidates for aggressive diuretic therapy.

Another biomarker which was found to be a significant finding in the symptoms unimproved group was elevated serum globulin comparatively. There are limited literature available on the prognostic value of increased serum globulin in HF patients. We found one study that reports it to serve as an inflammatory marker, appearing to play a part in the overall chronic inflammation process during HF. ${ }^{14}$ Patients not administered ACE inhibitors were prone for readmission $(>1)$ compared to other classes in HF. ACE inhibitors are an evidenced drug class reported to have good mortality benefit primarily prescribed to prevent ventricular remodeling. Previous studies have shown patients not prescribed with ACE inhibitors to be more prone for exacerbations of HF symptoms thus leading to increased readmissions and mortality. $11,15-17$

\subsection{Implications for practice}

It is advised to initiate an ACE inhibitor during the index hospitalization especially in Diabetics alongside effective medical management of hypertensive and hypothyroid patients as they are more susceptible to readmissions.

\subsection{Effective allied healthcare personnel led strategies to prevent $H F$ readmission}

Identifying effective strategies to reduce preventable HF readmissions would be valuable to patients, and healthcare providers. ${ }^{18} \mathrm{~A}$ recent meta-analysis concluded independent and combined effects of education and assessment to be beneficial strategies against readmissions of HF patients. ${ }^{19}$ Telehealth and phone care initiatives reduced congestive heart failure hospital readmission rate by $5 \%$, decreased costs, improved veteran satisfaction with overall care experience. ${ }^{20}$ Implementation of a team-based pharmacy practice model resulted in a significant decrease in the rate of 30-day readmissions for high-risk patients. $^{21}$

\subsection{Pharmaceutical care in improving HF outcomes}

HF therapeutic care usually takes place in more than one settings, contributed by a variety of healthcare providers. As patients transit 
from being inpatients to that making visits to ambulatory settings, multiple health care providers including pharmacists can play a significant role. The predominant areas of professional expertise include medication education and reconciliation, improve adherence to physician's recommendations and reduce medication errors. With a skill of effective communication with patients concerning their disease, medications and lifestyle, pharmacists can help identify barriers to medication adherence and resolving them. Importantly, education by clinical pharmacists should not be limited to patients but also encompass a health-care team comprising physicians, nurses, social workers, and caregivers. ${ }^{22}$ Other potential objectives during HF hospitalization and discharge are therapy optimization while dosing and drug selection, dosing titrated and aimed for maximum therapeutic effect primarily focusing on improvement of ejection fraction, patient stability, and overall quality of life. ${ }^{23}$ Recent studies reiterate positive HF outcomes by pharmacist interventions in reducing emergency department visits, and all-cause readmissions. ${ }^{24}$

\subsection{Nurses care in preventing HF readmissions}

Nurses play a vital role in prevention of hospital readmission among HF patients. Hospital based inpatient interventions peri-discharge are reported to result in better clinical outcomes and decrease HF readmissions. Trials have shown how a nurse-led multidisciplinary intervention focused towards providing comprehensive patient and family education, conducting medication review and nurse-led patient followup decreased readmissions by $56.2 \%$ with improved quality of life (QoL) scores. ${ }^{18}$ Another randomized controlled trial ${ }^{19}$ by cardiologist and HF nurse showed improvement various outcome measures like clinical outcomes, QoL, readmissions and morbidity. The intervention constituted intensive education, frequent monitoring, and close communication with patients' primary physician. Other nursing care intervention combining home health intensive nursing services with at least one outpatient follow-up within the week post hospital discharge is reported to reduce HF readmissions. ${ }^{20}$

\subsection{Medication adherence in preventing $H F$ readmissions}

Poor medication adherence is a common problem among HF patients leading to increased HF exacerbations, reduced physical function, readmission and mortality. Medication adherence should be addressed in regular follow-up visits, and evidenced based interventions to improve adherence should be a key part of HF self-care programs. ${ }^{25}$ Continuing patient and caregiver education about the importance of therapeutic compliance could play a vital role towards containing disease progression. ${ }^{26}$ Studies have shown patients' adherence to their prescribed HF medications had fewer HF symptoms and resulted in cardiac event-free survival. ${ }^{27}$

\subsection{Limitations}

There are few limitations to the study. Criteria on number of days (time frame) for readmissions were not set hence, included patients' rehospitalization were random. Re-hospitalization were not distinguished into emergency or ward visits. Further research is required to understand specific factors such as stages of the existing comorbidities or the severity of HF symptoms. Additionally, HF specific invasive biomarkers or possible lifestyle habits could be other potential factors predicting readmission or worsening clinical outcome needs to be further evaluated.

\section{Conclusions}

Our study re-establishes the fact about categorizing the patients based on comorbidities, importance of switching IV to PO diuretics and the importance of ACE inhibitors in reducing readmissions in HF. The significance of a comprehensive cardiac involving a multidisciplinary approach aiming cost effective therapy, medication education while discharge, personalized care in-hospital and home based for continuous follow-up could determine better morbidity and mortality prevention.

\section{Funding}

No funding was received for this study.

\section{Authors' contributions}

V. Kunhikatta, JPK Pathiraj, SSL Akkineni and R Chandrababu, conceived and designed the study. SSL Akkineni and O. Mohammed involved in data collection. SSL Akkineni, O. Mohammed and V. Kunhikatta conducted analysis and interpretation of data. SSL Akkineni and O. Mohammed drafted the manuscript. V. Kunhikatta, JPK Pathiraj, $\mathrm{R}$ Chandrababu, T. Devasia were involved in revising the manuscript critically for important intellectual content.

\section{Ethics approval and consent to participate}

This study was approved by the Institutional Ethical Committee of Kasturba Hospital, Manipal. (IEC 611/2017). The personal identification details of participants were not required at any stage during the study.

\section{Declaration of competing interest}

The author(s) declared no potential conflicts of interest with respect to the research, authorship, and/or publication of this article.

\section{Acknowledgements}

We would like to thank the Manipal Academy of Higher Education (MAHE), Manipal College of Pharmaceutical Sciences and the Department of Cardiology, Kasturba Medical College, Manipal for providing necessary facilities for carrying out this study.

\section{References}

1. Roger VL, Go AS, Lloyd-Jones DM, et al. Heart disease and stroke statistics-2011 update: a report from the American Heart Association. Circulation. 2011;123(4):e18-e209.

2. Rajadurai J, Tse HF, Wang CH, Yang NI, Zhou J, Sim D, Understanding the epidemiology of heart failure to improve management practices: an asia-pacific perspective. J Card Fail. 2017;23(4):327-339.

3. Mozaffarian D, Benjamin EJ, Go AS, et al. Heart disease and stroke statistics-2015 update: a report from the American Heart Association. Circulation. 2015;131(4):e29-322.

4. Blecker S, Paul M, Taksler G, Ogedegbe G, Katz S. Heart failure-associated hospitalizations in the United States. J Am Coll Cardiol. 2013;61(12):1259-1267.

5. Reyes EB, Ha JW, Firdaus I, et al. Heart failure across Asia: same healthcare burden but differences in organization of care. Int J Cardiol. 2016;223:163-167.

6. Thanusubramanian H, Kishore MK, Shetty R, Amberkar MB. A retrospective study of the treatment outcome of drug therapies used in heart failure patients with associated Co-morbidities in a tertiary care hospital. J Clin Diagn Res. 2016;10(6):FC06-9.

7. Wu JR, Lee KS, Dekker RD, et al. Prehospital delay, precipitants of admission, and length of stay in patients with exacerbation of heart failure. Am J Crit Care. 2016;26(1):62-69.

8. Greenberg BH, Abraham WT, Albert NM, et al. Influence of diabetes on character istics and outcomes in patients hospitalized with heart failure: a report from the organized program to initiate lifesaving treatment in hospitalized patients with heart failure (OPTIMIZE-HF). Am Heart J. 2007;154(2):277 e1-8.

9. Rodondi N, Newman AB, Vittinghoff E, et al. Subclinical hypothyroidism and the risk of heart failure, other cardiovascular events, and death. Arch Intern Med. 2005; 165(21):2460-2466.

10. Lombardi F, Belletti S, Battezzati PM, Lomuscio A. Acupuncture for paroxysmal and persistent atrial fibrillation: an effective non-pharmacological tool? World $J$ Cardiol. 2012;4(3):60-65.

11. Gheorghiade M, Pang PS. Acute heart failure syndromes. J Am Coll Cardiol. 2009;53(7):557-573.

12. Ponikowski P, Voors AA, Anker SD, et al. ESC Guidelines for the diagnosis and treatment of acute and chronic heart failure: the Task Force for the diagnosis and 
treatment of acute and chronic heart failure of the European Society of Cardiology (ESC)Developed with the special contribution of the Heart Failure Association (HFA) of the ESC. 2016 Eur Heart J. 2016;37(27) 2129-20.

13. Humbert M, Sitbon O, Yaici A, et al. Survival in incident and prevalent cohorts of patients with pulmonary arterial hypertension. Eur Respir J. 2010;36(3):549-555.

14. Gabay C, Kushner I. Acute-phase proteins and other systemic responses to inflammation. N Engl J Med. 1999;340(6):448-454.

15. Kostis WJ, Xie H, Sargsyan D. Predictors of readmission or death among patients who were discharged alive with a first diagnosis of heart failure in a statewide database. $J$ Am Coll Cardiol. 2018;71(11 Supplement):A855.

16. Maggioni AP, Anker SD, Dahlstrom U, et al. Are hospitalized or ambulatory patients with heart failure treated in accordance with European Society of Cardiology guidelines? Evidence from 12,440 patients of the ESC Heart Failure Long-Term Registry. Eur J Heart Fail. 2013;15(10):1173-1184.

17. Ziaeian B, Fonarow GC. The prevention of hospital readmissions in heart failure. Prog Cardiovasc Dis. 2016;58(4):379-385

18. Rich MW, Beckham V, Wittenberg C, Leven CL, Freedland KE, Carney RM. A multidisciplinary intervention to prevent the readmission of elderly patients with congestive heart failure. N Engl J Med. 1995;333(18):1190-1195.

19. Wan TTH, Terry A, Cobb E, McKee B, Tregerman R, Barbaro SDS. Strategies to modify the risk of heart failure readmission: a systematic review and meta-analysis. Health Serv Res Manag Epidemiol. 2017;4 2333392817701050.

20. Messina W. Decreasing congestive heart failure readmission rates within 30 Days at the tampa VA. Nurs Adm Q. 2016;40(2):146-152

21. Anderegg SV, Wilkinson ST, Couldry RJ, Grauer DW, Howser E. Effects of a hospitalwide pharmacy practice model change on readmission and return to emergency department rates. Am J Health Syst Pharm. 2014;71(17):1469-1479.

22. Sadik A, Yousif M, McElnay JC. Pharmaceutical care of patients with heart failure. $\mathrm{Br}$ $J$ Clin Pharmacol. 2005;60(2):183-193.

23. McNeely EB. Treatment considerations and the role of the clinical pharmacist throughout transitions of care for patients with acute heart failure. $J$ Pharm Pract. 2017;30(4):441-450.

24. Milfred-LaForest SK, Chow SL, DiDomenico RJ, et al. Clinical pharmacy services in heart failure: an opinion paper from the heart failure society of America and American College of clinical pharmacy Cardiology practice and research network. $J$ Card Fail. 2013;19(5):354-369.

25. Ruppar TM, Cooper PS, Mehr DR, Delgado JM, Dunbar-Jacob JM, et al. Medication adherence interventions improve heart failure mortality and readmission rates: systematic review and meta-analysis of controlled trials. J Am Heart Assoc. 2016;5(6):e002606.

26. Shah D, Simms K, Barksdale DJ, Wu JR. Improving medication adherence of patients with chronic heart failure: challenges and solutions. Res Rep Clin Cardiol 2015;6(1):87-95.

27. Wu JR, Moser DK. Medication adherence mediates the relationship between heart failure symptoms and cardiac event-free survival in patients with heart failure. $J$ Cardiovasc Nurs. 2018;33(1):40-46. 